\title{
VIRTUAL DIVING IN THE UNDERWATER ARCHAEOLOGICAL SITE OF CALA MINNOLA
}

\author{
F. Bruno ${ }^{\text {a,* }}$, A. Lagudi a ${ }^{\text {a L. Barbieri }}{ }^{\text {a, }}$ M. Muzzupappa ${ }^{\text {a }}$, M. Mangeruga ${ }^{\text {b }}$, F. Pupo ${ }^{\text {b }}$,

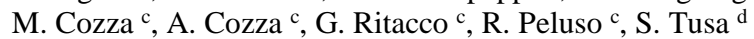 \\ a Department of Mechanical, Energy and Management Engineering (DIMEG), University of Calabria, \\ 87036 Rende (CS), Italy - (f.bruno, antonio.lagudi, loris.barbieri, muzzupappa)@unical.it \\ ${ }^{\mathrm{b}}$ Department of Electronics, Computer Science and System Sciences (DIMES), University of Calabria, \\ 87036 Rende (CS), Italy - (mangeruga, pupo)@unical.it \\ c 3DResearch S.r.l. \\ 87036 Rende (CS), Italy - (m.cozza, a.cozza, g.ritacco, r.peluso)@3dresearch.it \\ d Soprintendenza del Mare della Regione Sicilia \\ 90133 Palermo, Italy - sebtusa@archeosicilia.it
}

\section{Commission II}

KEY WORDS: Virtual Reality, virtual exploitation, underwater 3D reconstruction, Underwater Cultural Heritage

\begin{abstract}
:
The paper presents the application of the technologies and methods defined in the VISAS project for the case study of the underwater archaeological site of Cala Minnola located in the island of Levanzo, in the archipelago of the Aegadian Islands (Sicily, Italy). The VISAS project (http://visas-project.eu) aims to improve the responsible and sustainable exploitation of the Underwater Cultural Heritage by means the development of new methods and technologies including an innovative virtual tour of the submerged archaeological sites.

In particular, the paper describes the 3D reconstruction of the underwater archaeological site of Cala Minnola and focus on the development of the virtual scene for its visualization and exploitation. The virtual dive of the underwater archaeological site allows users to live a recreational and educational experience by receiving historical, archaeological and biological information about the submerged exhibits, the flora and fauna of the place.
\end{abstract}

\section{INTRODUCTION}

The Underwater Cultural Heritage (UCH) represents a vast historical and scientific resource of highest importance for understanding the development of human civilization and, as a consequence, a fundamental and essential source for the upbringing and education of the next human generations. In addition to this historical and educative role, the UCH also stands as an interesting opportunity for the tourism development of the coastal areas. In fact, underwater archaeological sites, be them shipwrecks or sunken cities, are considerably fascinating for the public, both for the sense of mystery that surrounds them and the symbiosis between the artifacts and the sea life.

Thus, the UCH has historical, cultural, social and environmental importance that offers opportunities for self-sustainment and, at the same time, contributes to the economic growth, promotion and development of the coastal areas but, in return, it needs protection, care and investment. To this end, considerable improvements are going on since the last decades especially thanks to an articulated implementation of the Unesco conventions 1970 (prevention of illicit traffic), 1972 (World Cultural and National Heritage), and 2001 (Underwater Cultural Heritage). In particular, the 2001 Unesco Convention on the Protection of the Underwater Cultural Heritage (Unesco 2001) has led to the definition of basic principles and recommendations, ratified by 51 states, for the protection and in-situ conservation of the submerged archaeological sites. These recommendations have encouraged the international collaboration among universities, research centers, and companies that has led to the running of a plethora of projects for the development of innovative methods and technologies for in-situ conservation (Bacpoles 2005; Gregory 2012; Gündoğdu et al. 2015; Bruno et al. 2016a, 2015a, 2015b) and promotion and exploitation (Chapman et al. 2008; Haydar et al. 2011; Varinlioğlu 2011) of the underwater archaeological sites. One of them is the VISAS project (Virtual and augmented exploitation of Submerged Archaeological Sites http://www.visas-project.eu) that is a collaborative research project funded by MIUR (Italian Ministry of Education, University and Research); it has started on $1^{\text {st }}$ April 2014 and ended on $30^{\text {th }}$ September 2016. It aims to implement an integrated package of services, based on the development of new methods and technologies, for improving the visitor experience and enjoyment of underwater archaeological sites (Bruno et al. 2016b). These services are intended, on the one hand, to promote diving tourism by improving the divers' experience in the underwater site and, on the other hand, to promote the induced tourist activity through the development of 
an innovative, educative and attractive virtual tour of the site (Bruno et al. 2016c).

The paper presents the application of the technologies and methods defined in the VISAS project for the case study of the underwater archaeological site of Cala Minnola. In particular, the paper describes the 3D reconstruction of the underwater archaeological site and focus on the development of the virtual scene for its visualization and interaction.

\section{ARCHAEOLOGICAL CONTEXT}

The underwater archaeological site of Cala Minnola is located on the East coast of the island of Levanzo, in the archipelago of the Aegadian Islands, few miles from the west coast of Sicily (figure 1). The site preserves the wreck of a Roman cargo ship at a depth from the sea level ranged from 25 to 30 meters.

The site is characterized by a complex morphology, in fact the shipwreck lies on a sandy seabed surrounded by a boulder field that rises until a depth of 12 meters. Furthermore, the area is covered by large seagrass beds of Posidonia and presents both strong surface and deep-sea currents.

The shipwreck was discovered around the 1970 and since then it has been subjected to frequent looting along the years. Finally, restoration and conservation activities, carried out in the summer of 2005, unveiled other remains covered by a layer of sand half a meter thick. The research activities went on for half a year and since the July of 2006 the archaeological site is monitored by means of a video surveillance system.

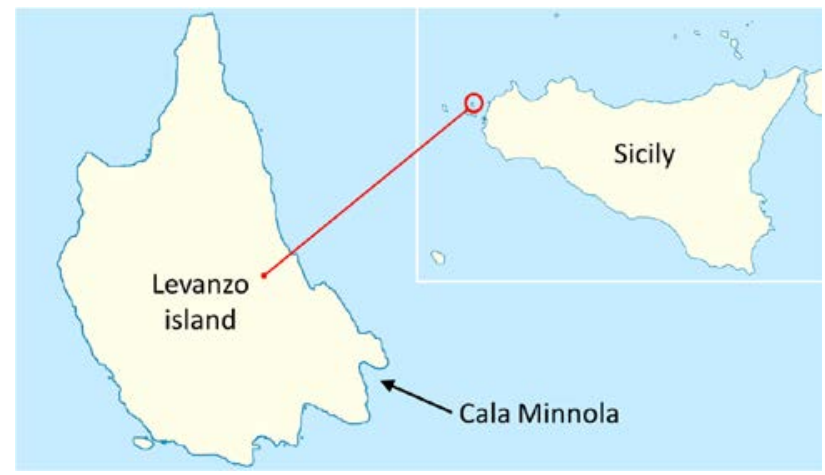

Figure 1. Geographical localization of the underwater archaeological site of Cala Minnola

The roman ship sunk off the coast of Cala Minnola bay in the first century B.C.. It was carrying hundreds of amphorae, of the Dressel 1b type, which should have been filled with wine. The ship belonged to the Papia, a wealthy roman family of shipowners and merchants that operated throughout the Mediterranean sea.

During the sinking, the vessel broke into two amidships and many pieces of the hull and amphorae were scattered across the seabed. Today, few are the traces of the wooden elements of the vessel, and only one lead pipe (belonging to the bilge pipe) is present. A great number of amphorae, about fifty, and fragments of black-painted pottery lies at a depth of 27 meters in the place where the stern sunk (figure 2).

The bow of the vessel sunk 50 meters far from the stern and its position has been identified thanks to the discovery of a large bower anchor.

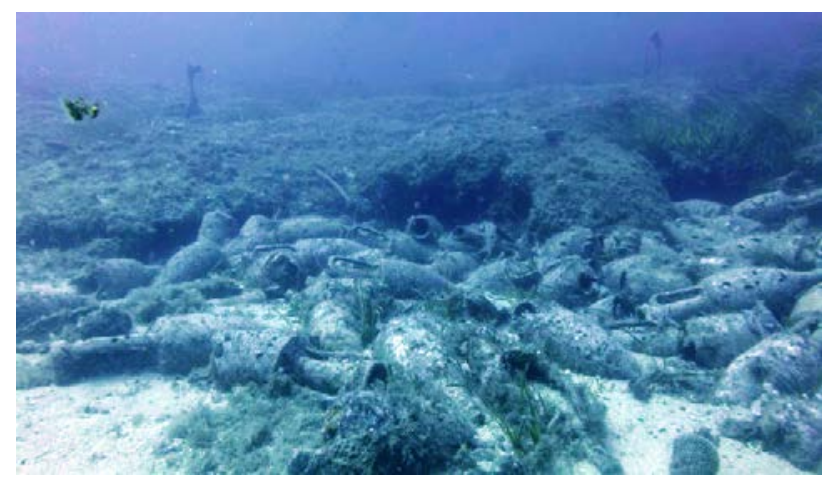

Figure 2. Submerged archaeological remains in the underwater site of Cala Minnola

\section{3D RECONSTRUCTION}

The 3D reconstruction of the submerged archaeological site of Cala Minnola has been a long and difficult process because of the underwater environment. In fact, on one hand, the depth of the underwater site and the strong sea currents of the area require advanced open diver certificates and skilled divers to perform the acquisition activities. On the other hand, the large size of the area and the different turbidity and brightness conditions required the adoption of both acoustic and optical 3D reconstruction techniques (Lagudi et al. 2016). In fact, while the acoustic techniques are usually adopted for acquiring a great amount of data at long distances even under bad visibility conditions, on the contrary, the optical techniques are more suited for close-range acquisitions and allow for gathering highresolution, accurate $3 \mathrm{D}$ data and textures, but the results are strongly influenced by turbidity. In particular, the acoustic survey has been carried out by means standard procedures by adopting a high frequency multibeam equipment, placed on a medium-sized boat, that allows to obtain the acoustic bathymetry of the seabed up to 100 meters depth from the sea level. The following image shows the acoustic bathymetry map of the archaeological site of Cala Minnola and the surrounding seabed.

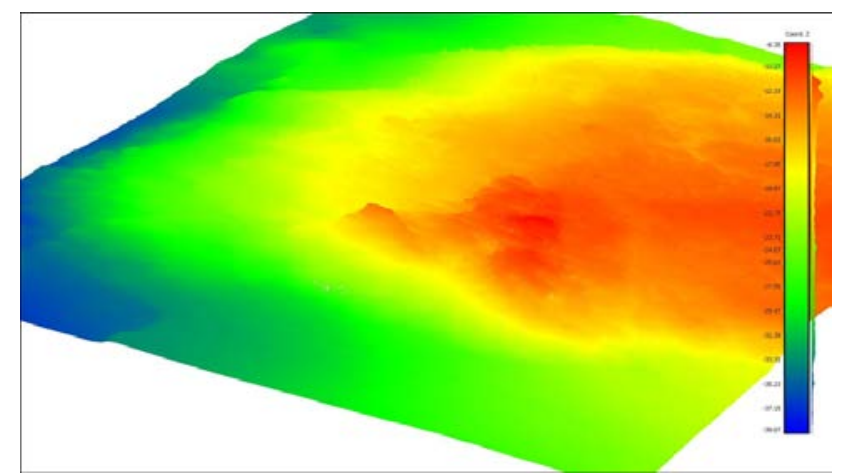

Figure 3. Acoustic bathymetry of the seabed in which the underwater archaeological site of Cala Minnola lays

The optical survey has been performed according to standard photogrammetric techniques that required two diver operators for three consecutive days, with one morning and one afternoon diving session. During the aerial photography layout (fig.4) the divers swam at an average distance of $1.5 \mathrm{~m}$ above the site, acquiring overlapping transects along straight lines that cover 
the whole area. Further acquisitions have been acquired using oblique poses in order to prevent occluded areas.

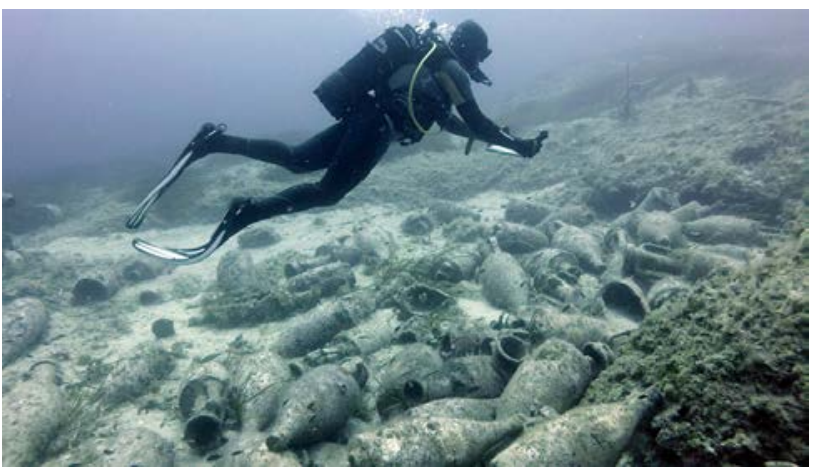

Figure 4. Optical acquisition tasks performed in the underwater archaeological site of Cala Minnola

Thanks to the optical survey a dataset of 1200 images has been created. Some image enhancement techniques have been applied on the dataset, in particular a color chart and external illuminating sources have been adopted in order to improve the chromatic quality of the pictures and faster the data processing time. The enhanced images have been then used to obtain the 3D model of archaeological area (fig.5).

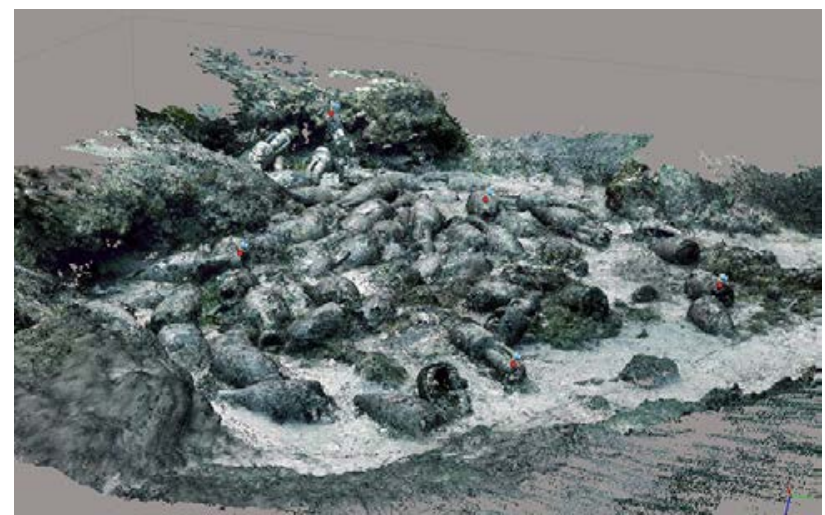

Figure 5. 3D point cloud obtained by processing the image data set

Subsequently, the optical and the acoustic meshes required alignment and merge operations. In particular, the location, orientation and scaling of the optical 3D models on the acoustic map have been performed thanks to the geolocation of some GPSs (Ground Control Points) performed by adopting an USBL system. In addition to the GPSs, the divers took several measurements by means of measuring tapes, rulers and targets in order to improve the accuracy of the final model. About the merging of the two different representations the operation has been performed by means a selective fitting of the acoustic mesh along the contours shared with the optical mesh. The result is a polygonal multiresolution mesh on which some manual refinements have been performed in order to correct geometry errors due to the complexity of the reconstructed scenario.

The last step consists in the texturing of the multiresolution mesh. In particular, techniques based on the projection and blending of 2D images on the 3D surface have been adopted to place textures on the portion of the mesh representing the archaeological remains. The remaining low-resolution polygon mesh of the seabed has been textured with a tile-based mapping approach that just requires to set same sample images instead of large textures.

The final texturized 3D model is depicted in the following figure.

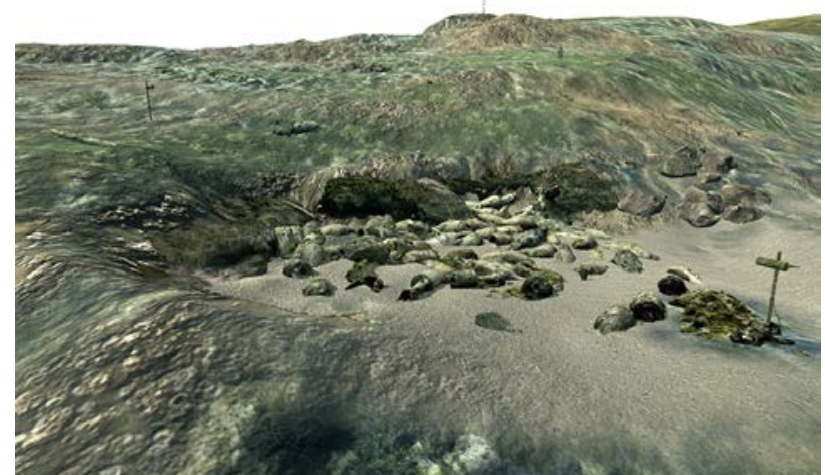

Figure 6. Final textured 3D model of the underwater archaeological site of Cala Minnola and the surrounding seabed

\section{SCENE CREATION}

Once the creation of the textured 3D model of the underwater archaeological area has been achieved, it has been adopted as starting point to build the immersive environment in which to perform the virtual diving experience. The creation of the scene has been carried out through an editing software specifically implemented in the cross-platform game engine Unity (Unity). The adoption of the Unity framework allows the programming of a software that can be used directly via web and communicate, by means of a web service software, with a database for the uploading and downloading of the data. The virtual scene has been populated with $3 \mathrm{D}$ models of the flora and fauna typical of the specific marine ecosystem, such as fishes, sponges, seagrass and seaweed plants. Furthermore, points of interest (POIs) have been added to the virtual scene in form of 3D large head map tacks (fig.7) which color depends by the category they belong to, e.g. yellow for the historical and archaeological information and green for biological ones.

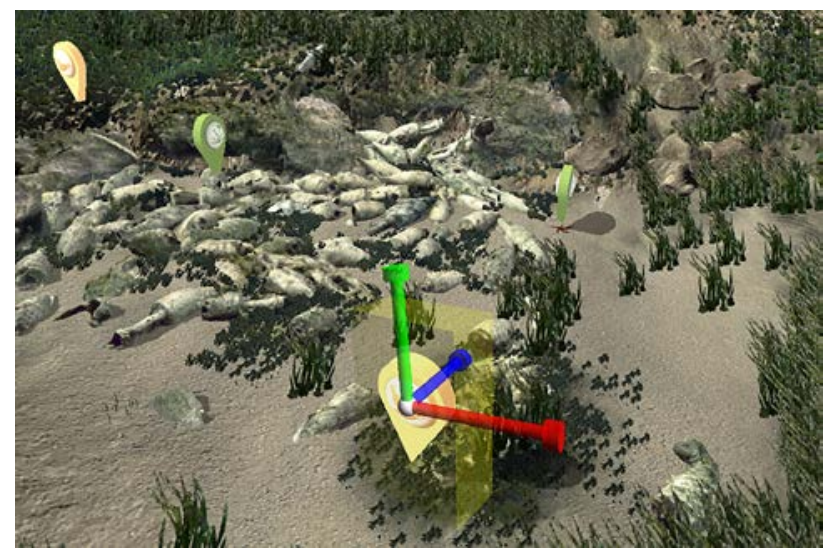

Figure 7. Adding a 3D object of a POI in the virtual scene

The POIs have been defined and geolocated during the optical and acoustic survey activities. They refer the location of interesting objects of specific feature types, such as one or a group of archaeological remains, flora or fauna. This information has been stored into the database in sheet format 
that includes their attributes (name, geographic coordinates, category/type) and multi-media data (text, image, audio and video).

In order to make the virtual underwater scenario more visually realistic some graphical effects have been applied, such as refractions, fog, caustics of the particulate, etc. (fig.8).

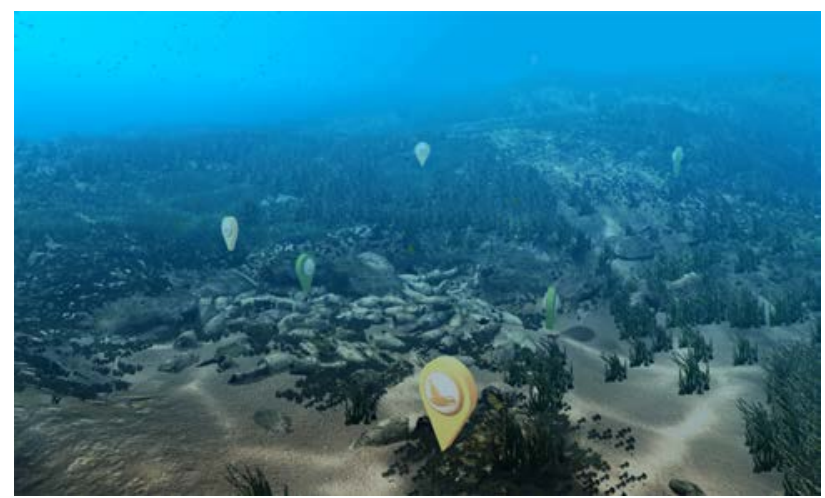

Figure 8. Virtual scene of the underwater archaeological site of Cala Minnola.

\section{FREE AND GUIDED DIVING TOUR}

The virtual diving of the underwater archaeological site of Cala Minnola can be performed according two different modalities: a free and a guided tour. The first mode allows users to freely dive in the underwater virtual scenario going up and down, backwards and forwards without any limit of time, he/she can interact with the POIs and enjoy of the flora and fauna typical of the site for as long as he/she likes.

In addition to the free exploration, the virtual diving features also a guided navigation system that allows users to simulate a real diving session taking into account the shortest path to safely visit the POIs of the underwater sites. This navigation mode allows to make users aware of certain time constraints and human body's limitations, arising from scuba diving related problems, typical of the underwater environment. This modality has been implemented developing a pathfinding algorithm that: recognizes obstacles within the $3 \mathrm{D}$ environment; generates the shortest paths through the POIs avoiding obstacles and minimizing the pressure at which the diver is subjected; verifies the generated paths' admissibility according to constraints imposed by the underwater environment. In particular, two constraints have been taken into account: the air in the diving cylinder and the decompression stops needed to complete the scuba diving session safely. If the constraints are not feasible then the path is not eligible, which means that the diver cannot visit all the waypoints in the remaining time. In this case, in order to find an admissible path, it is necessary to discard a waypoint at a time until the path becomes eligible, respecting in any case the priorities between the waypoints. The pathfinding feature has been developed on the basis of the $A^{*}$ searching algorithm (Hart et al. 1968) that constructs a tree of weighted paths starting from a specific node and expands the paths, one step a time, until one of them ends at a predetermined goal node. In order to achieve the goal the algorithm requires a partitioning of the search space that has been performed by means of a 3D grid.

The heuristic formula defined for the underwater environment takes into account the distance, weighted according to 1D, 2D or $3 \mathrm{D}$ movements, and the pressure conceived as a bonus and computed as the distance from the seabed. As depicted in figure
9, the path is represented by cubes, so that ones that lay at a greater depth have a higher cost than cubes with less depth. About the bonus, related to the pressure, it is computed taking into account the distance of the cubes from the seabed. This distance is calculated along the $\mathrm{z}$-axis of the grid where the origin lays in the deepest point of the seabed. The idea is to provide a bonus depth-related calculated from the $\mathrm{z}$ component of the cubes that represent the path $\left(b_{\text {press. }}=\sum z_{i}\right)$. Then the heuristic formula has been defined as follows: $H=w_{3 D} d_{3 D}+$ $w_{2 D} d_{2 D}+w_{1 D} d_{1 D}-b_{\text {press. }}$ where $w_{i}$ are the weights and $d_{i D}$ are the distance of the three movement types.

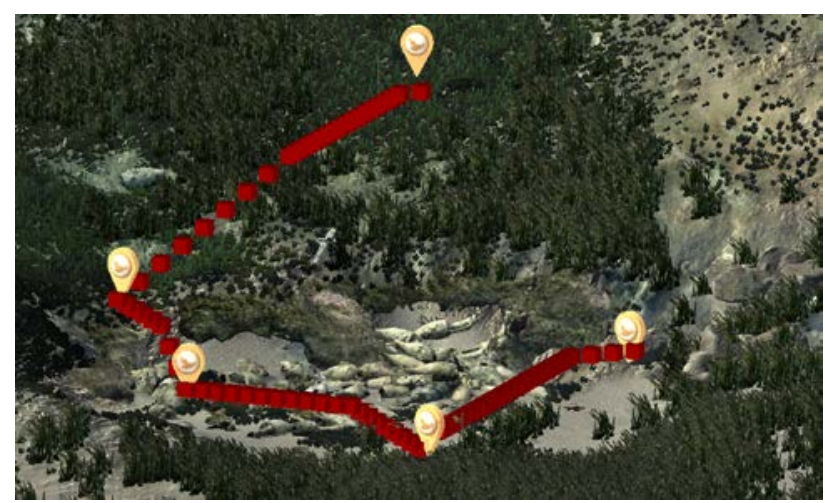

Figure 9. Pathfinding algorithm applied to the site of Cala Minnola

\section{VIRTUAL REALITY SETUP}

The virtual diving in the underwater archaeological site of Cala Minnola has been presented to the public on the $6^{\text {th }}$ November 2016 at the exhibition "Mirabilia Maris. Treasures from the seas of Sicily". The exhibit is dedicated to the historic underwater archaeological testimony of the Sicily, from prehistoric times to more recent years. It takes place inside the Norman Palace in Palermo (Italy) until the $6^{\text {th }}$ March 2017, after that the exhibition, already housed in Amsterdam and Oxford, will be hosted in the Ny Carlsberg Glyptotek museum in Copenhagen (Denmark) and the Landesmuseum museum of Bonn (Germany).

The setup of the virtual diving system has been designed on the basis of an user centered design (UCD) approach for VR systems (Barbieri et al., 2017) that allows to improve users' experience and make an easy to use user interface that can be easily understood and interpreted by a large variety of audiences, even by technologically naïve users.

In particular, different embodiments of the VR systems have been taken into account, such as monitors and projectors for the visualization and touch-screen consoles and trackballs for the interaction. After an accurate evaluation of the pros and cons of the different solutions, an HD monitor and a multi-touch tablet have been adopted for the user interaction and exploitation of the virtual diving.

About the touch-screen remote control it could be a handheld device, i.e., tablet, or fixed in a specific position. The first solution can usually be adopted when there is an operator that stands over the system and gives the controls to the visitors that want to enjoy the virtual exhibit. Instead, the second solution can be employed when the system is intended for unattended operation and, since the console cannot be moved, it is possible to increase the screen size of the touchscreen in order to enhance its legibility. The last solution as then been adopted for the development of the hardware architecture of the VR system. 
Since the virtual exhibit was intended to be used by many different audiences, such as middle and high school students, college students, tourists, etc., ergonomic studies have been performed too in order to find the optimal positioning of the visualization device and its control system. Also the grade of the touch-screen console has been studied in order to keep users' movements as natural as possible, with particular attention to the most repetitive ones, i.e. the neck and shoulder extension movements.

The following image shows the final setup of the virtual diving system.

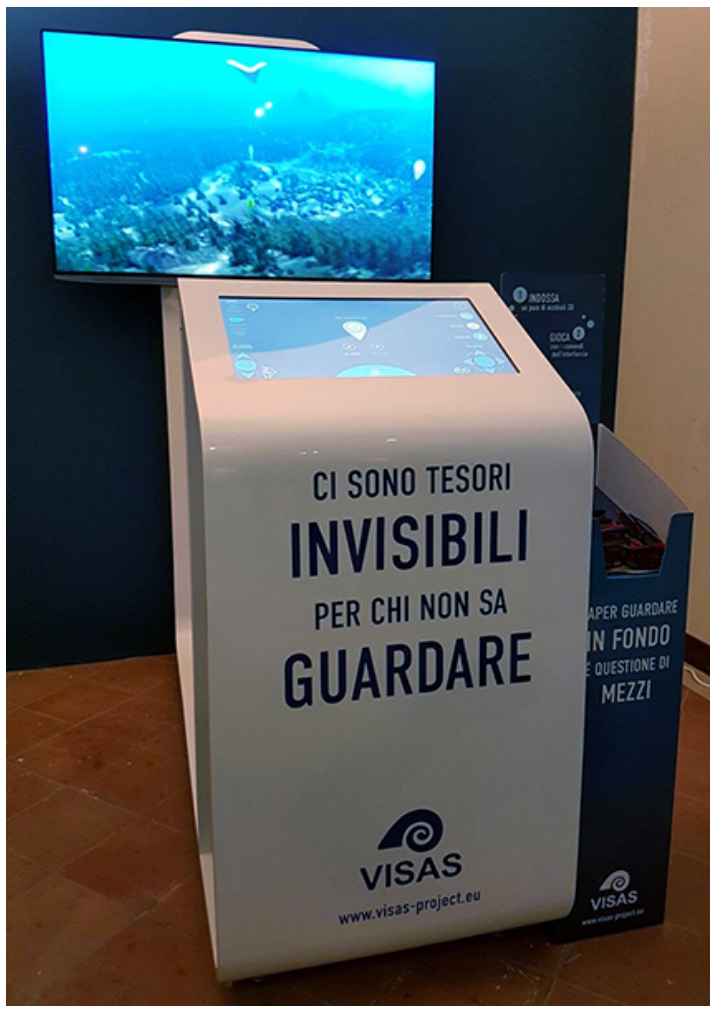

Figure 10. Final setup of the VR system

A 3D full HD monitor, based on passive technology, has been adopted in order to improve the visitors' sense of immersion and presence in the virtual environment (fig.11). The passive technology has been preferred to the active one because active 3D glasses are expensive and need batteries to work. Furthermore, passive 3D glasses are lighter and more comfortable.

The user interface displayed on the tablet provides all the input functionalities needed to explore the $3 \mathrm{D}$ environment and to get access to the multimedia data. In particular, the UI features two large command buttons, respectively, to go back and forth and to rotate the camera's point of view. On the top left side of the UI, a slider controls the depth of the camera view from the water surface. While, on the top right side, two circle buttons allow the user to switch between the guided tour modality and a free navigation mode.

When the user approaches a POI element the UI displays a visual warning and, at the same time, provides two command buttons that allow the user to question or skip it. In the first case, a new window appears in the centre of the UI containing textual, graphical and audio information related to the specific POI.
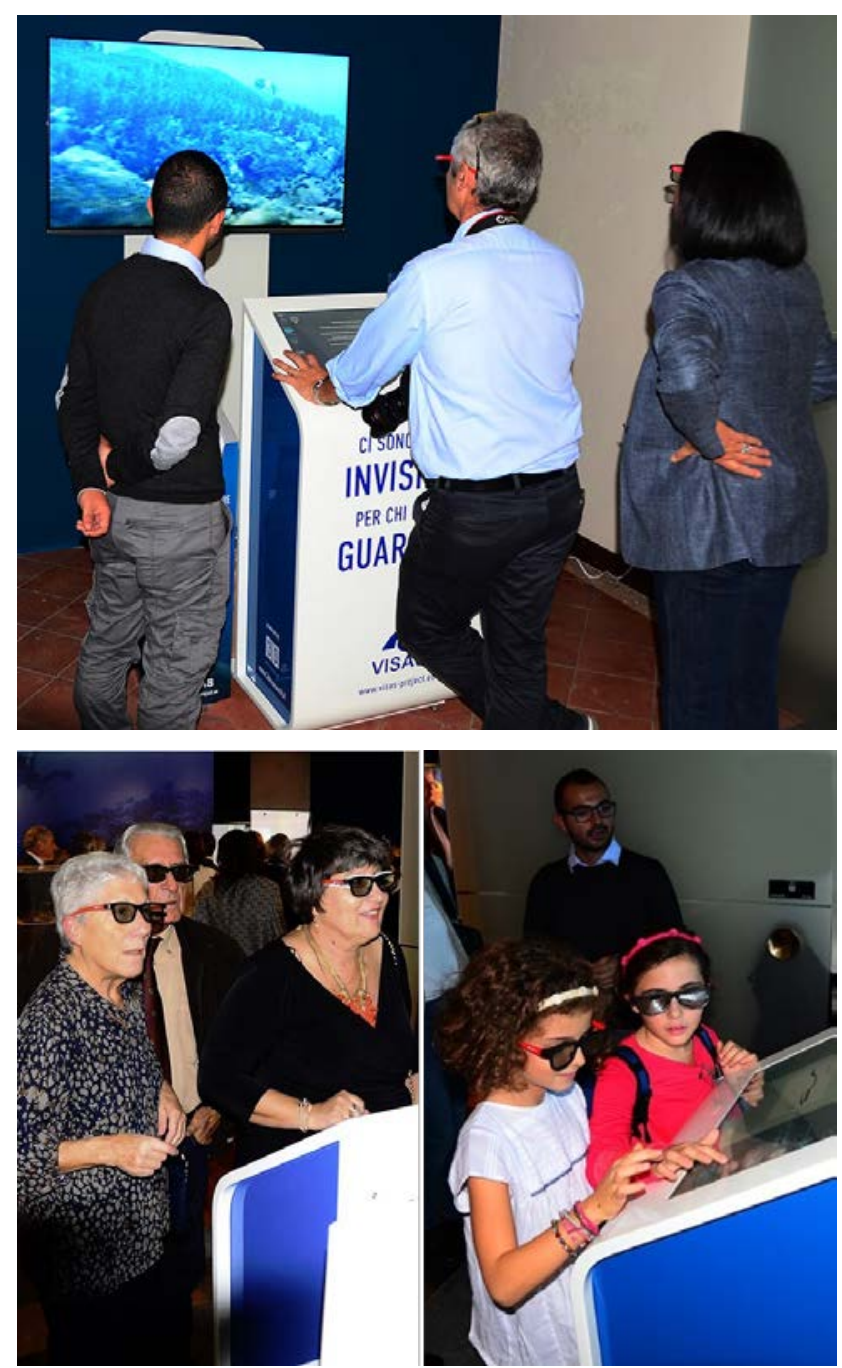

Figure 11. Visitors while experiencing the virtual diving in the underwater archaeological site of Cala Minnola

\section{CONCLUSIONS}

The paper has presented the application of the technologies and methods defined in the VISAS project for the case study of the underwater archaeological site of Cala Minnola. The virtual dive allows users to live a virtual experience inside the reconstructed 3D model of underwater archaeological site and provides a free or guided tour for the exploration of the various POIs that populate the archaeological area.

This virtual experience represents an innovative application of the VR technologies for the exploitation of the underwater cultural heritage. In fact, the virtual dive allows users to experience a virtual tour of the underwater site and interact with 3D objects in order to acquire historical, archaeological and biological information.

The VR exploitation of the underwater archaeological site of Cala Minnola is only one of the outcomes of the VISAS project that aims to develop an integrated set of methods and instruments for a more sustainable exploitation and a fruitful promotion of the underwater cultural heritage, both inside and outside of its original context of discovery. 


\section{ACKNOWLEDGEMENTS}

The VISAS Project (Ref. Start-Up PAC02L2-00040) has been financed by the Italian MIUR under the PAC Programme.

The authors would like to thank the Soprintendenza del Mare della Regione Sicilia for the permission to conduct the experimentation in the underwater archaeological site of Cala Minnola.

\section{REFERENCES}

Barbieri, L., Bruno, F., Mollo, F., Muzzupappa, M., 2017. User-centered design of a Virtual Museum system: a case study. International Joint Conference on Mechanics, Design Engineering and Advanced Manufacturing, Catania, Italy, Part I, pp. 155-165.

Bacpoles, 2005. Preserving cultural heritage by preventing bacterial decay of wood in foundation piles and archaeological sites. Final report BACPOLES-project (EVK4-CT-200100043), SHR, Wageningen Klaassen, R.K.W.M.

Bruno, F., Gallo, A., Barbieri, L., Muzzupappa, M., Ritacco, G., Lagudi, A., La Russa, M.F., Ruffolo, S.A., Crisci, G.M., Ricca, M., Comite, V., Davidde, B., Di Stefano, G., Guida, R., 2016a. The CoMAS project: new materials and tools for improving the in-situ documentation, restoration and conservation of underwater archaeological remains. Marine Technology Society (MTS) Journal 50(4), pp. 108-118(11).

Bruno, F., Lagudi, A., Muzzupappa, M., Lupia, M., Cario, G., Barbieri, L., Passaro, S., Saggiomo, R., 2016b. Project VISAS Virtual and augmented exploitation of Submerged Archaeological Sites: overview and first results. Marine Technology Society (MTS) Journal 50(4), pp. 119-129.

Bruno F., Lagudi A., Barbieri L., Muzzupappa M., Ritacco G., Cozza A., Cozza M., Peluso R., Lupia M., Cario G., 2016c. Virtual and Augmented Reality Tools to Improve the Exploitation of Underwater Archaeological Sites by Diver and Non-diver Tourists. Digital Heritage. Progress in Cultural Heritage: Documentation, Preservation, and Protection, Nicosia, Cyprus, Vol.10058, Part I, pp. 269-280.

Bruno F., Muzzupappa M., Gallo A., Barbieri L., Spadafora F., Galati D., Petriaggi B.D., Petriaggi R., 2015a. Electromechanical devices for supporting the restoration of underwater archaeological artefacts. Proceedings of MTS/IEEE OCEANS 2015 Conference, Genova (Italy), 18-21 May 2015.

Bruno F., Muzzupappa M., Lagudi A., Gallo A., Spadafora F., Ritacco G., Angilica A., Barbieri L., Di Stefano G., Guida R., Di Lecce N., Saviozzi G., Laschi C., 2015b. A ROV for supporting the planned maintenance in underwater archaeological sites. Proceedings of MTS/IEEE OCEANS 2015 Conference, Genova (Italy), 18-21 May 2015.

Chapman, P., Roussel, D., Drap, P., Haydar, M., 2008. Virtual Exploration of Underwater Archaeological Sites. Visualization and Interaction in Mixed Reality Environments, pp.141-148.

Gregory, D.J., 2012. Development of tools and techniques to survey, assess, stabilise, Monitor and preserve underwater archaeological sites. SASMAP. Euromed 2012 Proceedings, pp. 367-371.
Gündoğdu, H.T., Dede, M.İC., Taner, B., Ridolfî, A., Costanzi R., Allotta, B., 2015. An innovative cleaning tool for underwater soft cleaning operations. OCEANS 2015, Genova, pp. 1-8.

Hart, P. E., Nilsson, N. J., Raphael, B., 1968. A formal basis for the heuristic determination of minimum cost paths. IEEE transactions on Systems Science and Cybernetics, 4(2), pp.100107.

Haydar, M., Roussel, D., Maïdi, M., Otmane, S., Mallem, M., 2011. Virtual and augmented reality for cultural computing and heritage: a case study of virtual exploration of underwater archaeological site. Virtual reality, 15(4), pp. 311-327.

Lagudi, A., Bianco, G., Muzzupappa, M., Bruno, F., 2016. An Alignment Method for the Integration of Underwater 3D Data Captured by a Stereovision System and an Acoustic Camera. Sensors, 16(4), 536.

Unesco, 2001. Convention on the protection of the underwater cultural heritage, 2 November 2001. http://www.unesco.org

Unity. https://unity3d.com/

Varinlioğlu, G., 2011. Data Collection for a Virtual Museum on the Underwater Survey at Kaş, Turkey. International Journal of Nautical Archaeology, 40(1), pp.182-188. 